\title{
Acurácia de critérios para vagotonia no eletrocardiograma de repouso de 12 derivações: uma análise com curvas ROC
}

\author{
José Kawazoe Lazzoli1 ${ }^{1,3}$, Claudia Lúcia Barros de Castro ${ }^{5}$, \\ Antonio Claudio Lucas da Nóbrega ${ }^{2}$ e Claudio Gil Soares de Araújo ${ }^{4,5}$
}

\section{RESUMO}

Objetivo: Determinar a sensibilidade, a especificidade, os valores preditivos positivo e negativo e a acurácia de diferentes critérios eletrocardiográficos para vagotonia. Métodos: Foram avaliados 74 atletas olímpicos brasileiros (51 homens e 23 mulheres) com idade entre 16 e 42 anos ( $24 \pm 5$ anos), tendo sido realizados, entre outros exames, um ECG de repouso de 12 derivações e uma avaliação do tônus vagal cardíaco (TVC) pelo teste de exercício sem carga de quatro segundos (T4s), cujo índice B/C (indicativo da magnitude do TVC) foi utilizado como padrão-ouro. O T4s é um teste que se baseia no mecanismo da taquicardia inicial do exercício e foi anteriormente padronizado e validado como capaz de avaliar o TVC sem influência do componente simpático. Dados de 10 atletas foram excluídos devido à presença de critérios ecocardiográficos para hipertrofia ventricular esquerda, ainda que provavelmente fisiológica. Com o auxílio de curvas ROC (receiver operating characteristic), selecionou-se o ponto de corte com a melhor combinação entre sensibilidade (Sens) e especificidade (Esp) para cada variável do ECG, calculando-se os

\footnotetext{
1. Departamento de Morfologia da Universidade Federal Fluminense, Niterói, RJ, Brasil.

2. Departamento de Fisiologia e Farmacologia da Universidade Federal Fluminense, Niterói, RJ, Brasil.

3. Cor Diagnose/Ergocenter, Petrópolis, RJ, Brasil.

4. Programa de Pós-Graduação em Educação Física da Universidade Gama Filho, Rio de Janeiro, RJ, Brasil.

5. Clínica de Medicina do Exercício - Clinimex, Rio de Janeiro, RJ, Brasil. Recebido em: 11/1/02

Aceito em: 15/2/02
}

Endereço para correspondência:

Dr. Claudio Gil S. Araújo

Clínica de Medicina do Exercício

Rua Siqueira Campos, 93/101

22031-070 - Rio de Janeiro, RJ - Brasil

E-mail: cgaraujo@iis.com.br valores preditivos positivo (VPP) e negativo (VPN) e a acurácia (AC). Foram ainda correlacionados o índice B/C e as variáveis do ECG. Resultados: Há fraca associação entre o índice B/C e as variáveis do ECG, à exceção da duração do intervalo R-R $(r=0,353 ; P=0,004)$, que também apresentou curva ROC significativa $\left(\chi^{2}=0,863 ; P=0,002\right)$, sendo que o critério intervalo $R-R \geq 990 \mathrm{~ms}$ apresentou os seguintes resultados: Sens - 100\%; Esp - 73\%; VPP - 20\%; VPN$100 \%$; AC $-75 \%$. As variáveis do ECG que apresentaram pontos de corte com acurácia $>80 \%$ foram: amplitude da onda $\mathrm{T}$ em $\mathrm{V}_{5} \geq 8,0 \mathrm{~mm}$; amplitude da onda $\mathrm{T}$ em $\mathrm{V}_{6} \geq$ 7,0mm; amplitude da onda Q em $\mathrm{V}_{5} \geq 7,0 \mathrm{~mm}$; amplitude da onda $\mathrm{R} \mathrm{em} \mathrm{V}_{4} \geq 50,0 \mathrm{~mm}$; presença de supradesnível de ponto $\mathrm{J}$ em duas ou mais derivações; presença de supradesnível de segmento S-T em cinco ou mais derivações e presença de transição precordial rápida. Discussão e conclusão: $O$ desempenho dos critérios eletrocardiográficos em termos de VPP, VPN e AC foi muito limitado, com a exceção da duração do intervalo R-R. A combinação de outros critérios como amplitude da onda $\mathrm{Tem}_{5} \mathrm{eV}_{6}$, amplitude da onda $\mathrm{R} \mathrm{em} \mathrm{V}_{4}$ e presença de supradesnível de ponto $\mathrm{J}$ e do segmento $\mathrm{S}-\mathrm{T}$ em várias derivações pode auxiliar na predição de vagotonia.

Palavras-chave: Curvas ROC. Eletrocardiografia. Tônus vagal cardíaco. Teste de 4 segundos.

\section{ABSTRACT}

Accuracy of vagotonia criteria in the 12-lead resting electrocardiogram: analysis with ROC curves

Objective: To determine sensitivity (Sens), specificity (Spec), positive (PPV) and negative (NPV) predictive value, and accuracy of different electrocardiographic criteria for vagotonia. Methods: Seventy-four Olympic athletes (51 male and 23 female; age $=24 \pm 5$ years) underwent an evaluation consisting of a 12-lead resting electrocardiogram (ECG), and an assessment of cardiac vagal tone (CVT) by the 4-second cycling exercise testing (4-SET), with the $B / C$ index utilized as the gold standard. The 4-sET is a well- 
standardized and valid protocol for CVT assessment based on the mechanism of exercise-onset tachycardia regardless of the sympathetic component. Using ROC (receiver operating characteristic) curves, the authors selected the cutpoint with the best sensitivity (Sens)/specificity (Spec) ratio for each ECG variable, and calculated the positive (PPV) and negative predictive values (NPV), and accuracy (AC). The authors also correlated $B / C$ index with ECG variables. Results: $B / C$ index showed a poor association with $E C G$ variables, with the exception of $R-R$ interval $(\mathrm{r}=0.353 ; \mathrm{P}$ $=0.004)$, that also presented a significant ROC curve $\left(\chi^{2}=\right.$ $0.863 ; \mathrm{P}=0.002$ ), whereas the $\mathrm{R}-\mathrm{R}$ interval $\geq 990 \mathrm{~ms}$ criterion showed the following results: Sens - 100\%; Spec$73 \%$; VPP $-20 \%$; VPN $-100 \%$; AC $-75 \%$. The ECG variables that presented cut-points with accuracy $>80 \%$ were: $T$-wave amplitude in lead $V_{5} \geq 8.0 \mathrm{~mm}$; T-wave amplitude in lead $V_{6} \geq 7.0 \mathrm{~mm}$; $Q$-wave amplitude in $V_{5} \geq 7.0 \mathrm{~mm}$; $R$ wave amplitude in $V_{4} \geq 50.0 \mathrm{~mm}$; presence of J-point elevation in two or more leads; presence of S-T segment elevation in five or more leads, and presence of fast precordial transition. Discussion and conclusion: The performance of ECG criteria considering $P P V, N P V$, and AC was generally very poor, with the exception of the $R$ - $R$ interval. The combination of other criteria such as $T$-wave amplitude in leads $V_{5}$ and $V_{6}, R$-wave amplitude in $V_{4}$, and presence of J-point and $S$-T segment elevation in several leads should help predicting vagotonia.

Key words: Cardiac vagal tone. Electrocardiography. ROC curves. 4-s cycling test.

\section{INTRODUÇÃO}

Em 1936, Shipley e Hallaran ${ }^{1}$ descreveram, em indivíduos aparentemente saudáveis, alterações do segmento ST semelhantes a padrões eletrocardiográficos patológicos. Esse padrão eletrocardiográfico foi caracterizado por um supradesnível do segmento ST com concavidade superior e ondas $\mathrm{T}$ altas e pontiagudas, tendendo à simetria, principalmente nas derivações precordiais ${ }^{2-5}$. Essa variação do eletrocardiograma de repouso (ECG) encontrada em 2,5\% a $14 \%^{2}$ dos adultos sem evidências de doenças cardiovasculares ou extracardíacas tem sido denominada de "síndrome de repolarização precoce" ${ }^{4,6,7} \mathrm{e}$ tem sido associada à presença de vagotonia ${ }^{4,8,9}$. Não obstante a aparente benignidade desses achados, padrões semelhantes também podem ser observados em condições patológicas, tais como pericardite aguda ${ }^{2,5,7-9}$, insuficiência coronariana aguda ${ }^{5,7}$, hipercalemia ${ }^{7}$ e infarto agudo do miocárdio ${ }^{2,11,12}$.

Na década de 70, estudos experimentais demonstraram que tônus vagal cardíaco (TVC) diminuído, conjugado com tônus simpático aumentado - na presença de isquemia la- boratorialmente induzida - reduzia o limiar de fibrilação ventricular, facilitando a ocorrência de arritmias malignas ${ }^{13-}$ ${ }^{15}$. Por outro lado, evidências clínicas mais recentes apontam TVC elevado como um fator prognóstico benéfico importante em pacientes coronariopatas ${ }^{16-18}$. Ainda, diversos estudos indicam que a atividade vagal aumentada, tanto farmacologicamente ${ }^{19,20}$ quanto diretamente ${ }^{21,22}$, possui efeitos antiarrítmicos.

Além da freqüência com a qual se realiza o diagnóstico eletrocardiográfico de vagotonia na prática cardiológica cotidiana, é importante destacar a validade, o baixo custo e a acessibilidade do ECG como método complementar. Em 1994, contudo, Lazzoli et al. ${ }^{23}$ mostraram que não há consenso de critérios para o diagnóstico de vagotonia entre os cardiologistas participantes de um congresso nacional de arritmias cardíacas. Dessa forma, parece oportuno testar formalmente esses critérios contra um padrão-ouro de vagotonia.

Assim, o nosso objetivo foi o de determinar a sensibilidade, a especificidade, os valores preditivos positivo e negativo e a acurácia de diferentes critérios ao ECG para a identificação de vagotonia em um grupo de indivíduos adultos sadios.

\section{MATERIAL E MÉTODOS}

\section{Amostra}

Foram inicialmente avaliados 74 atletas olímpicos brasileiros, sendo 51 do sexo masculino (masc.) e 23 do feminino (fem.), com idade variando entre 16 e 42 anos (média \pm desvio padrão $=24 \pm 5$ anos), a apenas algumas semanas antes da realização dos Jogos Olímpicos. A amostra incluía vários atletas que lograram excelentes resultados e/ ou medalhas olímpicas. Como parte da avaliação médicofuncional antes dos Jogos Olímpicos, os atletas foram submetidos a: exame clínico detalhado, exames laboratoriais de sangue, fezes e urina e exames complementares, incluindo telerradiografia de tórax e ecodopplercardiograma bidimensional com Doppler colorido. Isso permitiu afastar causas que sabidamente pudessem alterar o traçado eletrocardiográfico. O consumo máximo de $\mathrm{O}_{2}$ foi estimado pelo nomograma de Åstrand-Rhyming a partir de um teste de exercício submáximo de seis minutos de duração realizado com carga constante (homens: 2 a 2,5watts $/ \mathrm{kg}$ de peso e mulheres: 1 a 1,5watts $/ \mathrm{kg}$ ) a uma freqüência fixa de 50rpm em cicloergômetro de frenagem mecânica (Monark, Brasil). Todos os exames foram realizados entre duas e quatro horas após a última refeição, com a exceção dos exames laboratoriais, após jejum de 12 horas. Nas 24 horas anteriores à avaliação, os atletas não ingeriram nenhum medicamento de ação cardiovascular ou neural. 
Dos 74 atletas avaliados, 10 foram retirados da análise (ciclismo - quatro masc.; remo - três masc.; natação - dois masc. e um fem.) por apresentar hipertrofia ventricular esquerda (HVE) ao exame ecocardiográfico, ainda que muito provavelmente de natureza fisiológica, uma vez que não foi encontrada nenhuma anormalidade estrutural cardíaca de natureza congênita ou adquirida no ecocardiograma. A distribuição dos 64 atletas (42 masc., 22 fem.) participantes deste estudo nas diversas modalidades pode ser observada na tabela 1 .

\begin{tabular}{lccc}
\hline \multicolumn{3}{c}{$\begin{array}{c}\text { TABELA 1 } \\
\text { Distribuição dos atletas segundo } \\
\text { sexo e modalidade desportiva }\end{array}$} \\
\hline \multicolumn{1}{c}{ Modalidade } & $\begin{array}{c}\text { Sexo } \\
\text { masculino }\end{array}$ & $\begin{array}{c}\text { Sexo } \\
\text { feminino }\end{array}$ & Total \\
Arco e flecha & 1 & & \\
Atletismo & 3 & 0 & 1 \\
Canoagem & 2 & 1 & 4 \\
Ciclismo & 4 & 0 & 2 \\
Esgrima & 4 & 0 & 4 \\
Ginástica olímpica & 1 & 0 & 4 \\
latismo & 6 & 0 & 1 \\
Judô & 6 & 0 & 6 \\
Luta olímpica & 2 & 2 & 8 \\
Nado sincronizado & 0 & 0 & 2 \\
Natação & 9 & 4 & 4 \\
Remo & 4 & 3 & 12 \\
Voleibol & 0 & 0 & 4 \\
Total & 42 & 12 & 12 \\
\hline
\end{tabular}

\section{Critérios eletrocardiográficos}

O ECG foi realizado em repouso, com as 12 derivações padronizadas pela literatura, em decúbito dorsal sem inclinação, após um período de repouso ao leito de pelo menos 10 minutos. Os traçados foram obtidos com velocidade de $25 \mathrm{~mm}$ por segundo e com a amplitude calibrada de tal forma que $10 \mathrm{~mm}$ equivalessem a $1,0 \mathrm{mV}$.

Foram medidas e avaliadas as seguintes variáveis quantitativas ao ECG: intervalo R-R (R-R); duração da onda P (P) em $\mathrm{D}_{\text {II }}$; duração do intervalo P-R (P-R) em $\mathrm{D}_{\text {II }}$; duração do QRS (QRS) em $\mathrm{D}_{\text {II }}$; duração do intervalo Q-T, medido em aVL, corrigido segundo a fórmula de Bazett (QTc); amplitude da onda $\mathrm{T}$ em $\mathrm{V}_{1}\left(\mathrm{TV}_{1}\right), \mathrm{V}_{2}\left(\mathrm{TV}_{2}\right), \mathrm{V}_{3}\left(\mathrm{TV}_{3}\right), \mathrm{V}_{4}\left(\mathrm{TV}_{4}\right), \mathrm{V}_{5}$ $\left(\mathrm{TV}_{5}\right)$ e $\mathrm{V}_{6}\left(\mathrm{TV}_{6}\right)$; soma das amplitudes das ondas $\mathrm{T}$ nas derivações precordiais (Ttot); amplitude da onda $\mathrm{Q}$ em $\mathrm{V}_{4}$ $\left(\mathrm{QV}_{4}\right), \mathrm{V}_{5}\left(\mathrm{QV}_{5}\right)$ e $\mathrm{V}_{6}\left(\mathrm{QV}_{6}\right)$; soma das amplitudes das ondas $\mathrm{Q}$ em $\mathrm{V}_{4}, \mathrm{~V}_{5}$ e $\mathrm{V}_{6}$ (Qtot); amplitude da onda $\mathrm{R} \mathrm{em} \mathrm{V}_{4}\left(\mathrm{RV}_{4}\right)$, $\mathrm{V}_{5}\left(\mathrm{RV}_{5}\right)$ e $\mathrm{V}_{6}\left(\mathrm{RV}_{6}\right)$; soma das amplitudes das ondas $\mathrm{R}$ em $\mathrm{V}_{4}, \mathrm{~V}_{5}, \mathrm{~V}_{6}$ (Rtot). Foram também avaliadas ainda algumas variáveis qualitativas: presença de ondas $\mathrm{T}$ apiculadas ( $\mathrm{T}$ -
A); presença de supradesnível de ponto J (SupJ), presença de ondas U (U); presença de supradesnível do segmento ST (SupsT), presença de ondas J (J); e presença de transição precordial rápida (TPR). Com a exceção desta última variável, que manteve a dicotomia presença/ausência, convertemos as demais variáveis discretas da escala nominal à ordinal, quantificando o número de derivações nas quais a característica estava presente.

A mensuração e a avaliação das variáveis eletrocardiográficas foram realizadas independentemente por dois dos autores, que são observadores experientes, com formação cardiológica e que não conheciam o resultado da avaliação do TVC. A resolução da leitura foi de $0,25 \mathrm{~mm}$. Os resultados foram determinados por média nas variáveis quantitativas e por concordância de ambos nas variáveis qualitativas.

\section{Avaliação do TVC}

O TVC foi avaliado através de um método desenvolvido, padronizado e validado por Araújo et al. ${ }^{24}$, denominado "teste de quatro segundos" (T4s). O T4s foi realizado em um cicloergômetro mecânico de membros inferiores da marca Monark, após o ajuste individual da altura do selim e de um repouso de pelo menos cinco minutos. Consistiu em pedalar rapidamente sem resistência (com "carga zero") do quarto ao oitavo segundo de uma apnéia inspiratória máxima de 12 segundos de duração. Com o auxílio de um monitor/registrador eletrocardiográfico de um canal foi obtido um traçado, onde foram medidas duas variáveis em milissegundos: B, que corresponde ao intervalo R-R imediatamente anterior ao exercício ou o primeiro do exercício, aquele que for maior; e $\mathbf{C}$, que corresponde ao menor intervalo R-R durante o exercício. Foi obtido, assim, um índice adimensional, o índice $\mathbf{B} / \mathbf{C}$, que corresponde à divisão dos dois intervalos R-R especificados, isto é, B por C. Este índice reflete a taquicardia inicial do exercício, cujo mecanismo já foi demonstrado corresponder a inibição vagal pura, sem interferência do sistema nervoso simpáti$\mathrm{co}^{24,25}$. Além disso, a realização eventual da manobra de Valsalva durante os quatro segundos de pedalagem não interfere na resposta cronotrópica e, conseqüentemente, no índice $\mathrm{B} / \mathrm{C}^{26}$. Um resultado do índice $\mathrm{B} / \mathrm{C}$ igual a 1,00 teoricamente equivaleria à ausência do TVC, já que corresponderia à ausência de aceleração da FC durante o exercício dinâmico.

A manobra foi realizada duas vezes para cada atleta, com um intervalo não inferior a dois minutos. Para a nossa análise, foi escolhido o maior valor de B/C dentre as duas manobras. Para a determinação de vagotonia, escolhemos o ponto de corte para o índice B/C de 2,00, que corresponde ao percentil 97,5 em nosso banco de dados ${ }^{27}$, portanto, o 
limite superior da normalidade estatística considerando um intervalo de confiança de $95 \%$.

\section{Análise estatística}

A aplicação do teste de Kolmogorov-Smirnov demonstrou que todas as variáveis apresentavam distribuição normal. Os resultados de cada variável foram comparados entre os grupos vagotônicos (aqueles com índice $\mathrm{B} / \mathrm{C} \geq 2,00$ ) e não-vagotônicos (com índice $\mathrm{B} / \mathrm{C}<2,00$ ) através do teste $t$ para amostras independentes ou do teste de Mann-Whitney no caso das variáveis descontínuas. O grau de associação entre o índice $\mathrm{B} / \mathrm{C}$ e as variáveis contínuas e ordinais do ECG foi determinado através do coeficiente de correlação linear de Pearson $(r)$ e a correlação não-paramétrica de Spearman-rank $(r s)$, respectivamente. Para a variável transição precordial rápida, a amostra foi dividida entre os que a apresentavam e os que não a apresentavam e os valores de $\mathrm{B} / \mathrm{C}$ comparados através do teste $t$ simples. Tais procedimentos foram realizados no programa SigmaStat 2.0 (Jandel Corporation, EUA).

Com o auxílio de curvas ROC (de Receiver Operating Characteristic), selecionamos as variáveis que apresentaram acurácia significativa para a detecção de vagotonia, identificadas quando mostravam uma área sob a curva ROC significativa, ou seja, quando diferia significativamente da reta de identidade. Independente do resultado da análise da área sob a curva ROC, identificamos o ponto de corte de cada variável do ECG que oferecia a melhor combinação de sensibilidade e especificidade com o auxílio dessas mesmas curvas ROC. Em seguida, calculamos os valores preditivo positivo (VPP) e negativo (VPN) e a acurácia (AC). Tal procedimento justifica-se pelo fato de que a área sob a curva ROC corresponde à acurácia total (considerando todos os valores daquela variável), a qual depende de uma combinação do VPP e o VPN, enquanto alguns pontos de corte específicos podem ter acurácia relevante, mesmo sem atingir uma área sob a curva significativa.

As variáveis são apresentadas na forma de média \pm erro padrão para as variáveis contínuas ou mediana (25\%-75\%) para as variáveis discretas. A elaboração e a análise através das curvas ROC foram realizadas no programa $\mathrm{Gra}$ phROC 2.0 (Kairisto \& Poola, Finlândia). O nível de significância foi arbitrado em 5\% para todos os procedimentos.

\section{RESULTADOS}

Os exames clínicos revelaram-se todos normais do ponto de vista cardiovascular e pulmonar, com exceção de alguns atletas que apresentaram sopros mesossistólicos, os quais a ecodopplercardiografia verificou serem de origem funcional. Os eletrólitos, a uréia e a creatinina se encontra- vam dentro dos limites considerados normais. O consumo máximo de $\mathrm{O}_{2}$ estimado nos atletas masculinos foi $59,2 \pm$ $15,9 \mathrm{ml} \cdot \mathrm{kg}^{-1} \cdot \mathrm{min}^{-1} \mathrm{e}$ nas atletas, $49,6 \pm 12,3 \mathrm{ml} \cdot \mathrm{kg}^{-1} \cdot \mathrm{min}^{-1}$. Dentre todos os 64 atletas, somente um não apresentou ao menos um achado do ECG de repouso considerado diferente do padrão normal. Todos estavam em ritmo sinusal, com exceção de um atleta que apresentou ritmo atrial direito baixo. $\mathrm{O}$ traçado de outro atleta mostrou bloqueio do ramo direito do $1^{\circ}$ grau. Os achados mais comuns foram: bradicardia sinusal $(\mathrm{n}=19)$, segmento P-R longo ou no limite da normalidade $(\mathrm{n}=6)$, segmento P-R curto sem onda delta ( $\mathrm{n}$ $=5$ ), ondas $\mathrm{R}$ de grande amplitude ( $>20 \mathrm{~mm} ; \mathrm{n}=38$ ); ondas $\mathrm{T}$ apiculadas em ao menos uma derivação $(\mathrm{n}=29)$, presença de onda $U(n=60)$, supradesnível de ponto $J(n=$ 47), supradesnível do segmento ST $(\mathrm{n}=30)$.

A avaliação do índice B/C, indicativo do TVC, revelou resultados no grupo $(n=64)$ de $1,52 \pm 0,32$, acima do normalmente encontrado para indivíduos desse grupo etário ${ }^{27}$. Quatro atletas (dois do judô, um masc. e um fem.; um ciclista e um iatista) tiveram um $\mathrm{B} / \mathrm{C} \geq 2,00$ e foram, portanto, considerados, para a nossa análise inicial, como "definitivamente vagotônicos". Conforme esperado, os valores do índice B/C foram maiores no grupo vagotônico $(2,44 \pm$ $0,12)$ quando comparados com os não-vagotônicos $(1,46 \pm$ $0,03 ; P=0,002)$. A média do $\mathrm{B} / \mathrm{C}$ no grupo com transição precordial precoce, quando comparada com o grupo sem esta característica, não apresentou diferença significativa $(P=0,89)$.

Somente a variável intervalo R-R apresentou diferença entre os grupos vagotônico e não-vagotônico e uma associação com o índice $\mathrm{B} / \mathrm{C}(r=0,35 ; P=0,0039)$. As demais variáveis não apresentaram diferença entre grupos ou correlação significativa (tabela 2).

Quanto à análise da área sob as curvas ROC, mais uma vez somente o intervalo R-R mostrou resultado significativo $\left(\chi^{2}=0,863 ; P=0,002\right.$; figura 1$)$. Os outros critérios apresentaram resultados entre $0,375(\mathrm{P}=0,999)$ e $0,756(P$ $=0,336$ ).

Independente do resultado da análise da área sob a curva ROC, identificamos o ponto de corte de cada variável do ECG que oferecia a melhor combinação de sensibilidade e especificidade com o auxílio destas mesmas curvas ROC. Os resultados dos VPP, VPN e da AC correspondentes são mostrados na tabela 3. Os pontos de corte escolhidos das seguintes variáveis mostraram acurácia $>80 \%$ : amplitude da onda $\mathrm{T}$ em $\mathrm{V}_{5} \geq 8,0 \mathrm{~mm}$; amplitude da onda $\mathrm{T}$ em $\mathrm{V}_{6} \geq$ 7,0mm; amplitude da onda $\mathrm{Q}$ em $\mathrm{V}_{5} \geq 7,0 \mathrm{~mm}$; amplitude da onda $R$ em $V_{4} \geq 50,0 \mathrm{~mm}$; presença de supradesnível de ponto $\mathrm{J}$ em duas ou mais derivações; presença de supradesnível de segmento S-T em cinco ou mais derivações e presença de transição precordial rápida. 
TABELA 2

Variáveis eletrocardiográficas nos indivíduos vagotônicos $(n=4)$ e não-vagotônicos

( $n=60$ ) comparados pelo teste $t$ simples ou teste de Mann-Whitney (variáveis

discretas) e seus respectivos coeficientes de correlação linear de Pearson

ou Sperman-rank (variáveis discretas) com o índice B/C

\begin{tabular}{|c|c|c|c|c|c|}
\hline Variável & $\begin{array}{c}\text { Vagotônicos } \\
\text { (Índice } B / C \geq 2,00 \text { ) }\end{array}$ & $\begin{array}{c}\text { Não-vagotônicos } \\
\text { (Índice } B / C<2,00 \text { ) }\end{array}$ & $\boldsymbol{P}$ & $\begin{array}{l}\text { Correlação (r ou } \\
\text { rs) vs. Índice B/C }\end{array}$ & $\boldsymbol{P}$ \\
\hline $\mathrm{R}-\mathrm{R}$ & $1.138 \pm 76$ & $878 \pm 23$ & 0,007 & 0,353 & 0,004 \\
\hline $\mathrm{P}$ & $92,5 \pm 2,5$ & $83,8 \pm 1,3$ & 0,090 & 0,134 & 0,292 \\
\hline P-R & $165,0 \pm 14,4$ & $146,7 \pm 4,1$ & 0,157 & 0,006 & 0,957 \\
\hline QRS & $77,5 \pm 7,5$ & $70,8 \pm 1,9$ & 0,375 & $-0,017$ & 0,893 \\
\hline QTc & $360,0 \pm 5,8$ & $365,3 \pm 4,2$ & 0,570 & $-0,118$ & 0,354 \\
\hline $\mathrm{TV}_{1}$ & $-1,6 \pm 0,4$ & $-0,1 \pm 0,3$ & 0,255 & 0,024 & 0,851 \\
\hline $\mathrm{TV}_{2}$ & $7,3 \pm 1,7$ & $6,0 \pm 0,4$ & 0,422 & 0,191 & 0,130 \\
\hline $\mathrm{TV}_{3}^{2}$ & $8,5 \pm 1,1$ & $6,5 \pm 0,4$ & 0,193 & 0,150 & 0,236 \\
\hline $\mathrm{TV}_{4}^{3}$ & $8,8 \pm 6,3$ & $6,3 \pm 0,4$ & 0,149 & 0,128 & 0,312 \\
\hline $\mathrm{TV}_{5}^{4}$ & $8,6 \pm 1,9$ & $5,5 \pm 0,3$ & 0,107 & 0,225 & 0,074 \\
\hline $\mathrm{TV}_{6}^{5}$ & $6,1 \pm 1,2$ & $4,1 \pm 0,2$ & 0,093 & 0,214 & 0,089 \\
\hline Ttot & $37,6 \pm 6,8$ & $28,3 \pm 1,5$ & 0,124 & 0,208 & 0,098 \\
\hline $\mathrm{QV}_{4}^{*}$ & - & - & - & $-0,184$ & 0,146 \\
\hline $\mathrm{QV}_{5}^{4}$ & $2,1 \pm 1,6$ & $0,9 \pm 0,1$ & 0,708 & 0,173 & 0,171 \\
\hline $\mathrm{OV}_{6}^{5}$ & $2,5 \pm 1,4$ & $1,1 \pm 0,1$ & 0,202 & 0,225 & 0,073 \\
\hline Qtot & $4,6 \pm 2,9$ & $2,6 \pm 0,4$ & 0,204 & 0,104 & 0,415 \\
\hline $\mathrm{RV}_{4}$ & $25,6 \pm 8,6$ & $19,4 \pm 0,9$ & 0,153 & 0,070 & 0,579 \\
\hline $\mathrm{RV}_{5}^{4}$ & $30,9 \pm 11,8$ & $17,1 \pm 0,8$ & 0,166 & 0,220 & 0,081 \\
\hline $\mathrm{RV}_{6}^{5}$ & $23,0 \pm 7,3$ & $13,7 \pm 0,6$ & 0,117 & 0,204 & 0,105 \\
\hline Rtot & $79,5 \pm 27,7$ & $50,2 \pm 2,3$ & 0,292 & 0,169 & 0,182 \\
\hline $\mathrm{T}-\mathrm{A}$ & $1,5(0-3,5)$ & $0(0-3)$ & 0,835 & 0,047 & 0,707 \\
\hline SupJ & $2,5(2-4,5)$ & $2,0(1-4)$ & 0,551 & 0,125 & 0,325 \\
\hline$U$ & $4,0(3-7,5)$ & $3,5(3-4)$ & 0,367 & 0,117 & 0,356 \\
\hline SupST & $1(0-3,5)$ & $0(0-3)$ & 0,824 & $-0,075$ & 0,553 \\
\hline $\mathrm{J}$ & $0(0-0,5)$ & $0(0-0)$ & 0,889 & $-0,132$ & 0,298 \\
\hline
\end{tabular}

* - Nenhum atleta no grupo "vagotônico" apresentou ondas $Q$ na derivação $V_{4}$.

R-R: intervalo R-R; P: duração da onda P; P-R: duração do intervalo P-R; QRS: duração do QRS; QTc: duração do intervalo Q-T corrigido; TV1: amplitude da onda $T$ em $V_{1} ;$ TV2: amplitude da onda $T$ em $V_{2} ;$ TV3: amplitude da onda $T_{\text {em }} \mathrm{V}_{3}$; TV4: amplitude da onda T em $\mathrm{V}_{4}$; TV5: amplitude da onda $\mathrm{T}$ em $\mathrm{V}_{5}$; TV6: amplitude da onda $\mathrm{T}$ em $\mathrm{V}_{6}$; Ttot: soma das amplitudes das ondas $\mathrm{T}$ nas derivações precordiais; QV4: amplitude da onda $\mathrm{Q}$ em $\mathrm{V}_{4}$; $\mathrm{QV} 5$ : amplitude da onda $\mathrm{Q}$ em $\mathrm{V}_{5}$ QV6: amplitude da onda $\mathrm{Q}$ em $\mathrm{V}_{6}$; $\mathrm{Qtot}$ : soma das amplitudes das ondas $Q$ em $V_{4}, V_{5}$ e $V_{6}$; RV4: amplitude da onda $R$ em $V_{4}$, RV5: amplitude da onda $R$ em $V_{5}$ RV6: amplitude da onda $R$ em $V_{6} ; R$ tot: soma das amplitudes das ondas $R$ em $V_{4}, V_{5}$ e $V_{6} ; T-A$ : presença de ondas $T$ apiculadas; SupJ: presença de supradesnível de ponto J; U: presença de ondas U; SupST: presença de supradesnível do segmento S-T; J: presença de ondas J. As unidades das medidas são: milissegundos ( $\mathrm{ms}$ ) para a duração das ondas e intervalos; milímetros $(\mathrm{mm})$ para a amplitude das ondas; freqüência de ocorrência (f) em número de derivações para as variáveis discretas categóricas. Variáveis em média \pm EP para as variáveis contínuas ou mediana (25\%-75\%) para as variáveis discretas.

\section{DISCUSSÃO E CONCLUSÃO}

A literatura médica internacional vem mostrando a importância prognóstica de TVC elevado em indivíduos coronariopatas ${ }^{16-18}$. Contudo, a estratégia para quantificação do TVC permanece um tema em aberto. O ECG de repouso é um método amplamente disponível que poderia fornecer uma opção para a avaliação do TVC. Dessa forma, o presente estudo procurou validar critérios do ECG de repouso para detecção de vagotonia.

O padrão eletrocardiográfico conhecido como "síndrome de repolarização precoce" pode possuir várias características: 1) ondas J proeminentes ${ }^{4,7}$; 2) segmento ST supradesnivelado com concavidade superior ${ }^{2-6,10,11,28} ; 3$ ) ondas $\mathrm{T}$ relativamente altas e pontiagudas e, freqüentemente, simétricas ou tendendo à simetria ${ }^{2-5}$; 4) ocasionalmente, ondas $\mathrm{T}$ invertidas ${ }^{4}$; 5) ondas $\mathrm{Q}$ iniciais proeminentes e estreitas nas derivações precordiais esquerdas ${ }^{4} ; 6$ ) ondas $\mathrm{R}$ altas nas derivações precordiais esquerdas ${ }^{2,4,7} ; 7$ ) ondas $U$ proeminentes nas derivações precordiais intermédias ${ }^{2,4} ; 8$ ) transição precordial rápida ${ }^{4,7}$; 9) tendência à rotação anti-horária $\left.{ }^{4,7} ; 10\right)$ bradicardia sinusal ou frequiência cardíaca no limite inferior da faixa de normalidade ${ }^{4}$. Vários diferentes mecanismos foram propostos para essas alterações encontradas em $2,5 \%{ }^{4}$ a $14 \%^{2}$ dos adultos jovens aparentemente saudáveis. Um dos mecanismos mais comentados é uma antecipação do processo de repolarização ventricular ${ }^{3,6,10-}$ 


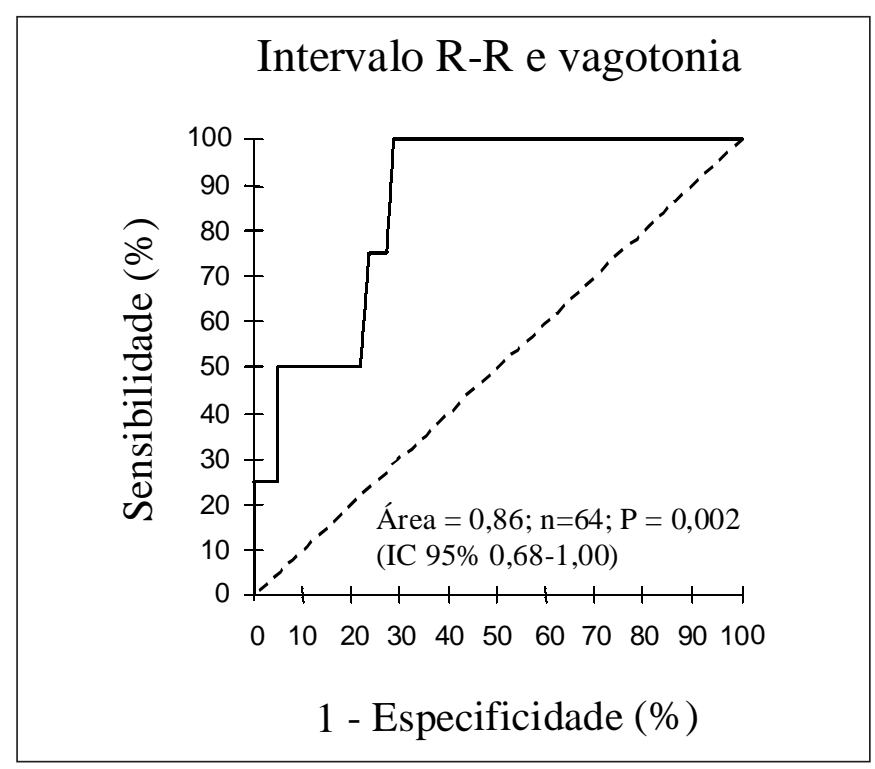

Fig. 1 - Curva ROC do critério intervalo $R$-R para predição de vagotonia, considerada presente com um ponto de corte de $B / C$ de 2,0 no teste de exercício quatro segundos $(n=64)$

${ }^{12,28}$, que já tem início enquanto determinadas áreas do ventrículo ainda se estão despolarizando. Entretanto, tais comentários são meramente especulativos e essa teoria é contestada por Mirvis ${ }^{29}$.

Vários autores também creditam ao TVC elevado a causa subjacente dessas alterações ao ECG da "síndrome de repolarização precoce" $", 30$. Da mesma forma, tais afirmações também são na sua maioria especulativas; os estudos experimentais existentes contêm falhas metodológicas que comprometem sua validade interna, como inclusão de indivíduos com doenças cardiovasculare ${ }^{28}$ e extracardía$\operatorname{cas}^{2,28,31}$, doses inadequadas de atropina de acordo com Jose e Taylor ${ }^{32}$ para produzir um bloqueio parassimpático completo $^{2,28,33}$, utilização de poucas derivações ${ }^{31,33}$ e, finalmente, nenhum dos autores por nós citados neste parágrafo exerceu um controle adequado das outras variáveis clínicas que sabidamente podem influenciar o traçado eletrocardiográfico. Em adição, não foi utilizado nenhum instrumento para avaliação e controle do TVC. O diagnóstico eletrocardiográfico de vagotonia permanecia de tal forma especulativo, que um estudo realizado com 40 cardiologistas presentes ao 9ํㅡㄹ Congresso Brasileiro de Arritmias Cardíacas (São José do Rio Preto, SP, dezembro de 1992), número que incluiu acima de dois terços dos palestrantes e apresentadores de temas-livres no Congresso, demonstrou claramente a falta de concordância quanto aos sinais do ECG que devem ser valorizados para o diagnóstico de vagotonia, visto que os 40 especialistas citaram espontaneamente 35 diferentes critérios ${ }^{23}$.
TABELA 3

Representação dos valores percentuais de sensibilidade (Sens), especificidade (ESP), valor preditivo positivo (VPP), valor preditivo negativo (VPN) e acurácia (AC) dos diferentes critérios do ECG para identificação de vagotonia. $O$ ponto de corte do índice $B / C$ utilizado foi de 2,00. As melhores combinações de Sens e ESP foram determinadas através de curvas ROC, sendo em seguida o VPP, VPN e AC calculados para os pontos de corte selecionados

\begin{tabular}{|c|c|c|c|c|c|}
\hline Critério & Sens & Esp & VPP & VPN & AC \\
\hline $\mathrm{R}-\mathrm{R} \geq 990 \mathrm{~ms}$ & 100 & 73 & 20 & 100 & 75 \\
\hline$P \geq 90 \mathrm{~ms}$ & 100 & 55 & 13 & 100 & 57 \\
\hline $\mathrm{P}-\mathrm{R} \geq 160 \mathrm{~ms}$ & 75 & 63 & 12 & 97 & 64 \\
\hline $\mathrm{QRS} \geq 90 \mathrm{~ms}$ & 50 & 82 & 15 & 96 & 79 \\
\hline $\mathrm{QTc} \leq 370 \mathrm{~ms}$ & 100 & 42 & 10 & 100 & 61 \\
\hline $\mathrm{TV}_{1} \geq 2,5 \mathrm{~mm}^{*}$ & 25 & 62 & 4 & 93 & 59 \\
\hline $\mathrm{TV}_{2} \geq 8,0 \mathrm{~mm}$ & 75 & 68 & 14 & 98 & 69 \\
\hline $\mathrm{TV}_{3} \geq 8,0 \mathrm{~mm}$ & 75 & 68 & 14 & 98 & 69 \\
\hline $\mathrm{TV}_{4} \geq 8,0 \mathrm{~mm}$ & 75 & 73 & 16 & 98 & 73 \\
\hline $\mathrm{TV}_{5}^{4} \geq 8,0 \mathrm{~mm}$ & 75 & 87 & 27 & 97 & 86 \\
\hline $\mathrm{TV}_{6} \geq 7,0 \mathrm{~mm}$ & 75 & 88 & 30 & 98 & 84 \\
\hline Ttot $\geq 39,0 \mathrm{~mm}$ & 75 & 80 & 20 & 98 & 79 \\
\hline $\mathrm{QV}_{4}^{* *}$ & - & - & - & - & - \\
\hline $\mathrm{QV}_{5}^{4} \geq 7,0 \mathrm{~mm}$ & 25 & 100 & 100 & 95 & 95 \\
\hline $\mathrm{QV}_{6} \geq 1,5 \mathrm{~mm}$ & 75 & 65 & 13 & 98 & 63 \\
\hline Qtot $\geq 2,0 \mathrm{~mm}$ & 75 & 53 & 9 & 97 & 55 \\
\hline $\mathrm{RV}_{4} \geq 50,0 \mathrm{~mm}$ & 25 & 100 & 100 & 95 & 95 \\
\hline $\mathrm{RV}_{5}^{4} \geq 22,0 \mathrm{~mm}$ & 75 & 77 & 18 & 98 & 77 \\
\hline $\mathrm{RV}_{6} \geq 18,0 \mathrm{~mm}$ & 75 & 80 & 20 & 98 & 79 \\
\hline Rtot $\geq 58,0 \mathrm{~mm}$ & 75 & 65 & 13 & 98 & 66 \\
\hline $\mathrm{T}-\mathrm{A} \geq 3$ deriv. & 50 & 67 & 9 & 95 & 66 \\
\hline SupJ $\geq 2$ deriv. & 100 & 52 & 9 & 100 & 96 \\
\hline Onda $U \geq 5$ deriv. & 50 & 78 & 13 & 96 & 77 \\
\hline SupST $\geq 5$ deriv. & 25 & 98 & 50 & 95 & 94 \\
\hline TPR (presença) & 25 & 95 & 25 & 95 & 91 \\
\hline Onda $\mathrm{J} \geq 1$ deriv. & 25 & 83 & 9 & 94 & 79 \\
\hline
\end{tabular}

Obs.: * - Foram consideradas ondas $\mathrm{T}$ positivas e negativas, acima de $2,5 \mathrm{~mm}$ de amplitude.

** - Nenhum atleta no grupo "vagotônico" apresentou ondas $Q$ na derivação $\mathrm{V}_{4}$

R-R: intervalo R-R; P: duração da onda P; P-R: duração do intervalo P-R; QRS: duração do QRS; QTc: duração do intervalo Q-T corrigido; TV1: amplitude da onda T em $V_{1} ;$ TV2: amplitude da onda $T$ em $V_{2} ;$ TV3: amplitude da onda $T$ em $V_{3} ; T V 4$ : amplitude da onda $T$ em $V_{4} ;$ TV5: amplitude da onda T em $V_{5} ;$ TV6: amplitude da onda $\mathrm{T}$ em $\mathrm{V}_{6}$; Ttot: soma das amplitudes das ondas $\mathrm{T}$ nas derivações precordiais: QV4: amplitude da onda $\mathrm{Q}$ em $\mathrm{V}_{4}$; OV5: amplitude da onda $\mathrm{Q}$ em $\mathrm{V}_{5}$ QV6: amplitude da onda $\mathrm{Q}$ em $\mathrm{V}_{6}$; Otot: soma das amplitudes das ondas $\mathrm{Q}$ em $\mathrm{V}_{4}, \mathrm{~V}_{5}$ e $\mathrm{V}_{6}$; RV4: amplitude da onda $R$ em $V_{4}$, RV5: amplitude da onda $R$ em $V_{5} R V 6$ : amplitude da onda $\mathrm{R}$ em $\mathrm{V}_{6}$; Rtot: soma das amplitudes das ondas $\mathrm{R}$ em $\mathrm{V}_{4}, \mathrm{~V}_{5}$ e $\mathrm{V}_{6}$; T-A: presença de ondas T apiculadas; SupJ: presença de supradesnível de ponto J; U: presença de ondas U; SupST: presença de supradesnível do segmento S-T; J: presença de ondas J; TRP: transição precordial rápida; deriv.: derivações.

No presente estudo, tivemos a oportunidade de avaliar uma amostra propositadamente viesada para incluir indivíduos vagotônicos em proporção maior que na população de indivíduos sadios não-atletas e na qual uma grande gama de dados clínicos e exames laboratoriais e complementares obtidos concomitantemente estava disponível. Em aden- 
do, tivemos a preocupação de virtualmente afastar toda e qualquer variável clínica que pudesse sabidamente alterar o traçado eletrocardiográfico. Todos os indivíduos eram assintomáticos, com exames clínicos sem nenhuma anormalidade que denotasse a presença de qualquer doença cardiovascular ou extracardíaca. O ecocardiograma afastou qualquer anormalidade estrutural congênita do coração que, embora assintomática, pudesse alterar o ECG; 10 atletas que apresentaram hipertrofia ventricular esquerda (HVE) foram excluídos da análise, mesmo não havendo indícios de HVE patológica, já que a HVE é um estado que pode alterar o ECG. Os eletrólitos, a uréia e a creatinina foram normais em todos os atletas. Nenhum dos atletas apresentou alterações de ECG de esforço que pudessem levar à suspeita de coronariopatia. Naturalmente, não realizamos um estudo cinecoronariográfico para afastar por completo a possibilidade de doença arterial coronariana (DAC) obstrutiva; mas levando em conta que todo o grupo era composto por atletas de elite, em nível de seleção olímpica (conseqüentemente, com um padrão muito diferenciado de exercício físico regular), todos assintomáticos do ponto de vista cardiovascular, e que todos se encontram na categoria de baixo risco cardiovascular do American College of Sports Medicine ${ }^{34}$, com probabilidade pós-teste extremamente baixa, podemos considerar a possibilidade de DAC desprezível.

O T4s é um teste que se acha padronizado e validado na literatura ${ }^{24-26}$ e baseia-se no mecanismo fisiológico de taquicardia inicial do exercício, que sabidamente se deve à inibição parassimpática, sem qualquer contribuição simpática. A resposta da FC na transição repouso-exercício tem características diferentes daquela durante a fase do exercício continuado. Na transição repouso-exercício a FC responde de forma tudo-ou-nada, analogamente ao potencial de ação de uma célula muscular ou neural. Desse modo, tal resposta não guarda relação com a intensidade do esforço, mas com o tônus vagal em repouso, servindo como fundamento teórico para utilização da resposta da FC no início do exercício como estratégia para quantificação do tônus vagal cardíaco. Dos testes de avaliação autonômica cardiovascular disponíveis na literatura, o T4s é um dos poucos a avaliar puramente o TVC, visto que na maioria dos outros testes há contribuições em graus variáveis de ambos os componentes do sistema nervoso autônomo ${ }^{35,36}$. Outros grupos também têm utilizado o T4s para avaliar o $\mathrm{TVC}^{37}$. Por ser um teste suficientemente validado na literatura, pela sua simplicidade, e por fornecer uma variável contínua para avaliação do TVC - o índice B/C - escolhemos o T4s para a nossa análise.

Na tabela 2, ficou clara a fraca associação entre o índice B/C e as variáveis eletrocardiográficas, com exceção do 56 intervalo R-R, que atingiu uma correlação significativa. Essa fraca associação entre o instrumento utilizado para avaliação do TVC e o ECG pode ser interpretada de diferentes formas: 1) os critérios do ECG por nós analisados, muitos dos quais são utilizados empiricamente para identificar vagotonia, não possuem o poder discriminativo que antes se pensava; 2) as características do ECG de repouso não possuem validade suficiente para determinação de TVC aumentado, em parte devido à complexidade intrínseca da avaliação da função autonômica cardiovascular e à dificuldade de separar as influências simpáticas e parassimpáticas; 3) existe pouca dispersão dos dados eletrocardiográficos na nossa amostra, fato que compromete sabidamente o poder estatístico da análise de correlação ${ }^{38}$.

A análise das curvas ROC mostrou que somente o intervalo R-R possui poder discriminativo significativo para detecção de vagotonia. Entretanto, com o auxílio das mesmas curvas ROC, selecionamos o ponto de corte que apresentou a melhor combinação de sensibilidade e especificidade, para cada variável eletrocardiográfica. E, nesse ponto de corte selecionado, calculamos ainda o VPP, o VPN e a AC. No caso do intervalo R-R o melhor ponto de corte foi $990 \mathrm{~ms}$, correspondente a uma freqüência cardíaca de $61 \mathrm{bpm}$. Como podemos observar na tabela 3 , há algumas variáveis do ECG que, apesar de não possuírem área sob a curva ROC significativa como o intervalo R-R, apresentam acurácia $>80 \%$ e, portanto, clinicamente útil, como amplitude da onda $\mathrm{T}$ em $\mathrm{V}_{5} \geq 8,0 \mathrm{~mm}$; amplitude da onda $\mathrm{T}$ em $\mathrm{V}_{6} \geq 7,0 \mathrm{~mm}$; amplitude da onda $\mathrm{Q}$ em $\mathrm{V}_{5} \geq 7,0 \mathrm{~mm}$; amplitude da onda $\mathrm{R}$ em $\mathrm{V}_{4} \geq 50$,0mm; presença de supradesnível de ponto $\mathrm{J}$ em duas ou mais derivações; presença de supradesnível de segmento S-T em cinco ou mais derivações e presença de transição precordial rápida. Curiosamente, muitos dos critérios descritos na literatura como indicativos de vagotonia apresentaram desempenho muito baixo.

Outra questão relevante, ao nosso ver, é o conceito de normalidade estatística, habitualmente utilizado para fixar vários critérios clínicos de "normalidade". Utilizamos como padrão-ouro para vagotonia o nível de B/C correspondente ao percentil 97,5 de uma série previamente publicada ${ }^{27-34}$, equivalente a 2,00; entretanto, nada nos garante que é justamente a partir de algum desses pontos de corte que haverá mais alterações ao ECG, ou que o limiar de FV estará particularmente reduzido. Podemos fazer uma analogia com a hipertrofia ventricular esquerda (HVE); o padrão-ouro clinicamente mais utilizado atualmente para HVE é a ecocardiografia e o critério mais aceito nos nossos dias para o diagnóstico de HVE corresponde justamente ao percentil 97,5 de uma amostra composta por 225 indivíduos, publicado em um artigo de Devereux et al., em 1984; nada, po- 
rém, nos assegura que é a partir exatamente desse ponto de corte que haverá mais alterações ao ECG, que o risco cardiovascular aumentará, ou que haverá maior prejuízo da função diastólica; provavelmente, o "ponto de corte" para que tais efeitos ocorram é diferente do ponto de corte estatístico (o percentil 97,5). Esse raciocínio pode ser extrapolado para a nossa análise. É possível que a "quantidade" de TVC necessária para alterar o ECG de repouso seja refletida por um valor diferente do B/C. Em outras palavras, a análise do presente trabalho refere-se especificamente à identificação de intensa vagotonia e pode não ser aplicável à detecção de níveis mais sutis de TVC aumentado.

Quando analisamos a tabela 3 podemos verificar que mesmo os critérios que possuem boa combinação de sensibilidade e especificidade apresentam VPP baixos e os VPN são de regra muito altos. Os dois critérios que contam com um VPP alto (QV5 $\geq 7,0 \mathrm{~mm}$ e RV4 $\geq 50,0 \mathrm{~mm})$ só o têm porque a curva ROC indicou um ponto de corte em um nível bastante alto (ambos os 100\% de VPP correspondem a 1/1), o que é ilustrado pela sensibilidade prejudicada. Esse fato é provavelmente influenciado pela desigualdade numérica acentuada entre o grupo "vagotônico" e o "não va-

\section{REFERÊNCIAS}

1. Shipley RA, Hallaran WR. The four-lead electrocardiogram in two hundred normal men and women. Am Heart J 1936;11:325-45.

2. Grusin H. Peculiarities of the African's electrocardiogram and the changes observed in serial studies. Circulation 1954;9:860-7.

3. Myers GB, Klein HA, Stofer BE, Hiratzka T. Normal variations in multiple precordial leads. Am Heart J 1947;34:785-808.

4. Schamroth L. The 12 lead electrocardiogram. Oxford: Blackwell, 1989: 26-9,162,179,183,188,243,248,299,338,345,414.

5. Thomas J, Harris E, Lassiter G. Observations on the T wave and S-T segment changes in the precordial electrocardiogram of 320 young $\mathrm{Ne}$ gro adults. Am J Cardiol 1960;5:468-72.

6. Alimurung BN, Gilbert CA, Felner JM, Schlant RC. The influence of early repolarization variant on the exercise electrocardiogram: a correlation with coronary arteriograms. Am Heart J 1980;99:739-45.

7. Kambara H, Phillips J. Long-term evaluation of early repolarization syndrome (normal variant RS-T segment elevation). Am J Cardiol 1976; 38:157-61.

8. Hanne-Paparo N, Wendkos MH, Brunner D. T wave abnormalities in the electrocardiograms of top-ranking athletes without demonstrable organic heart disease. Am Heart J 1971;81:743-7.

9. Somers K, Rankin AM. The electrocardiogram in healthy East African (Bantu and Nilotic) men. Br Heart J 1962;24:542-8.

10. Fenichel NN. A long term study of concave RS-T elevation - A normal variant of the electrocardiogram. Angiology 1962;13:360-6.

11. Goldman MJ. RS-T segment elevation in mid- and left precordial leads as a normal variant. Am Heart J 1953;46:817-20.

12. Osher HL, Wolff L. Electrocardiographic pattern simulating acute myocardial injury. Am J Med Sci 1953;226:451-5.

13. Lown B, Verrier RL. Neural activity and ventricular fibrillation. N Engl J Med 1976; 294:1165-70. gotônico" (dos 64 atletas analisados, apenas quatro ficaram no grupo dos "vagotônicos"), quando se considera como ponto de corte para o índice B/C o valor de 2,00.

\section{CONCLUSÃO}

Dessa forma, podemos concluir que alguns critérios eletrocardiográficos possuem níveis satisfatórios e equilibrados de sensibilidade e especificidade, VPP, VPN e AC para a identificação de um determinado nível de vagotonia, como intervalo R-R, TV5, TV6, QV6, RV5 e onda U. A maioria dos critérios utilizados na literatura alcançou valores bastante baixos. Ainda, tais critérios atingem melhor desempenho com níveis mais altos de TVC, mas não é possível determinar outro ponto de corte além do estatístico para diagnosticar "vagotonia". É muito provável que um estudo que seja realizado com indivíduos aparentemente saudáveis, com bom controle das variáveis clínicas que possam influir no traçado eletrocardiográfico, e envolvendo o bloqueio farmacológico temporário completo do TVC, possa identificar de modo objetivo e consistente as consequiências no traçado eletrocardiográfico da presença do TVC.

14. Lown B, Verrier RL. Neural factors and sudden death. In: Schwartz PJ, Brown AM, Malliani A, Zanchetti A, editors. Neural mechanisms in cardiac arrhythmias - Perspectives in cardiovascular research. Vol. 2. New York: Raven Press, 1978:87-98.

15. Verrier RL, Lown B. Sympathetic-parasympathetic interactions and ventricular electrical stability. In: Schwartz PJ, Brown AM, Malliani A, Zanchetti A, editors. Neural mechanisms in cardiac arrhythmias - Perspectives in cardiovascular research. Vol. 2. New York: Raven Press, 1978:75-86.

16. Kleiger RE, Miller JP, Bigger Jr JT, Moss AJ. Multicenter Postinfarction Research Group: Heart rate variability: a variable predicting mortality following acute myocardial infarction. Am J Cardiol 1987;59:25662.

17. LaRovere MT, Bigger Jr JT, Marcus FI, Mortara A, Schwartz PJ. Baroreflex sensitivity and heart-rate variability in prediction of total cardiac mortality after myocardial infarction. Lancet 1998;351:478-84.

18. Bonaduce D, Petretta M, Marciano F, et al. Independent and incremental prognostic value of heart rate variability in patients with chronic heart failure. Am Heart J 1999;138:273-84.

19. De Ferrari GM, Vanoli E, Curcuruto P, Tommasini G, Schwartz PJ. Prevention of life-threatening arrhythmias by pharmacologic stimulation of the muscarinic receptors with oxotremorine. Am Heart J 1992;124:88390.

20. De Silva RA, Verrier RL, Lown B. The effects of psychological stress and vagal stimulation with morphine on vulnerability to ventricular fibrillation (VF) in the conscious dog. Am Heart J 1978;95:197-203.

21. Myers RW, Pearlman AS, Hyman RM, et al. Beneficial effect of vagal stimulation and bradycardia during experimental acute myocardial ischemia. Circulation 1974;49:943-7.

22. Vanoli E, De Ferrari GM, Stramba-Badiale M, Hull SS Jr, Foreman RD, Schwartz PJ. Vagal stimulation and prevention of sudden death in con- 
scious dogs with a healed myocardial infarction. Circ Res 1991;68:147181.

23. Lazzoli JK, Annarumma MO, Araújo CGS. Critérios para o diagnóstico eletrocardiográfico de vagotonia - Existe consenso na opinião de especialistas? Arq Bras Cardiol 1994;63:377-81.

24. Araújo CGS, Nóbrega ACL, Castro CLB. Heart rate responses to deep breathing and 4-seconds of exercise before and after pharmacological blockade with atropine and propranolol. Clin Auton Res 1992;2:35-40.

25. Nóbrega ACL, Castro CLB, Araújo CGS. Relative roles of the sympathetic and parasympathetic systems in the 4-s exercise test. Braz J Med Biol Res 1990;23:1259-62.

26. Nóbrega AC, Williamson JW, Araújo CGS, Friedman DB. Heart rate and blood pressure responses at the onset of dynamic exercise: effect of Valsalva manoeuvre. Eur J Appl Physiol 1994; 68:336-40.

27. Araújo CGS, Pegado BPC. Vagal tone estimation in 10-s resting EKG tracings: a validation study using the 4 -s exercise testing. Med Sci Sports Exerc 2000;32(Suppl):S146 (abstract).

28. Chelton LG, Burchell HB. Unusual RT segment deviations in electrocardiograms of normal persons. Am J Med Sci 1955:230:54-60.

29. Mirvis DM. Evaluation of normal variations in S-T segment patterns by body surface isopotential mapping: S-T segment elevation in absence of heart disease. Am J Cardiol 1982;50:122-8.

30. Bedford DE, Thomas G. The sickle-shaped R-T plateau, a common RST pattern in health. Br Heart J 1954;16:469.
31. Wendkos MH. The influence of autonomic imbalance on the human electrocardiogram. Am Heart J 1944;28:549-67.

32. Jose AD, Taylor RR. Autonomic blockade by propranolol and atropine to study intrinsic myocardial function in man. J Clin Invest 1969;48: 2019-31.

33. Dauchot P, Gravenstein JS. Effects of atropine on the electrocardiogram in different age groups. Clin Pharmacol Ther 1971;12:274-80.

34. American College of Sports Medicine. ACSM's Guidelines for exercise testing and prescription. $6^{\text {th }}$ ed. Philadelphia: Lippincott Williams and Wilkins, 2000:137-64.

35. Castro CLB, Nóbrega ACL, Araújo CGS. Testes autonômicos cardiovasculares - Uma revisão crítica - Parte I. Arq Bras Cardiol 1992;59:7585 .

36. Castro CLB, Nóbrega ACL, Araújo CGS. Testes autonômicos cardiovasculares - Uma revisão crítica - Parte II. Arq Bras Cardiol 1992;59: 151-7.

37. Knopfli B, Bar-Or O. Vagal activity and airway response to ipratropium bromide before and after exercise in ambient and cold conditions in healthy cross-country runners. Clin J Sports Med 1999;9:170-6.

38. Edwards AL. Factors influencing the magnitude of the correlation coefficient. In: An introduction to linear regression and correlation. $2^{\text {nd }} e d$. New York: WH Freeman, 1984:35-47. 